CLINICAL STUDY

\title{
Nocturnal intermittent hypoxia as an associated risk factor for microalbuminuria in Japanese patients with type 2 diabetes mellitus
}

\author{
Shinya Furukawa ${ }^{1}$, Isao Saito ${ }^{2}$, Shin Yamamoto ${ }^{1}$, Teruki Miyake ${ }^{1}$, Teruhisa Ueda $^{1}$, Tetsuji Niiya ${ }^{4}$, \\ Masamoto Torisu $^{5}$, Teru Kumagi ${ }^{1}$, Takenori Sakai ${ }^{6}$, Hisaka Minami ${ }^{7}$, Hiroaki Miyaoka ${ }^{8}$, Susumu Sakurai ${ }^{9}$, \\ Bunzo Matsuura ${ }^{1}$, Morikazu Onji ${ }^{1}$ and Takeshi Tanigawa ${ }^{3}$ \\ Departments of ${ }^{1}$ Gastroenterology and Metabology ${ }^{2}$ Department of Basic Nursing and Health Science and ${ }^{3}$ Public Health, Ehime University Graduate \\ School of Medicine, Shitsukawa, Toon, Ehime 791-0295, Japan, ${ }^{4}$ Department of Internal Medicine, Matsuyama City Hospital, Ehime, Japan, ${ }^{5}$ Department \\ of Internal Medicine, Saiseikai Saijo Hospital, Ehime, Japan, ${ }^{6}$ Department of Internal Medicine, Yawatahama General City Hospital, Ehime, Japan, \\ ${ }^{7}$ Department of Internal Medicine, Ehime Niihama Hospital, Ehime, Japan, ${ }^{8}$ Department of Internal Medicine, Saiseikai Matsuyama Hospital, Ehime, Japan \\ and ${ }^{9}$ Department of Clinical Laboratory Science, Tenri Health Care University, Nara, Japan \\ (Correspondence should be addressed to S Furukawa; Email: shinfuru@m.ehime-u.ac.jp)
}

\begin{abstract}
Objective: We estimated the prevalence of nocturnal intermittent hypoxia, a surrogate marker of obstructive sleep apnoea, among type 2 diabetes mellitus (T2DM) patients and examined the association between nocturnal intermittent hypoxia and microvascular diseases.

Design and methods: We recruited 513 Japanese patients (292 men and 221 women) with T2DM. Nocturnal intermittent hypoxia was diagnosed using the 3\% oxygen desaturation index, with less than five events per hour corresponding to normal and five events or more per hour corresponding to nocturnal intermittent hypoxia.

Results: The prevalence of nocturnal intermittent hypoxia was $45.4 \%$ among T2DM patients. The nocturnal intermittent hypoxia group was older and had a higher BMI, greater weight change since the age of 20 years, higher smoking rate and increased prevalence of hypertension, hyperlipidaemia, microalbuminuria and macroalbuminuria. Microalbuminuria (model 1: odds ratio (OR), 3.41; 95\% CI, 1.85-6.40; model 2: OR, 3.69; 95\% CI, 1.85-7.59 and model 3: OR, 3.12; 95\% CI, 1.45-6.95) and nephropathy (model 1: OR, 4.51; 95\% CI, 1.58-15.1; model 2: OR, 7.31; 95\% CI, 2.11-31.6 and model 3: OR, 5.23; 95\% CI, 1.45-23.8) were derived as factors from all the three statistical models and constantly associated with nocturnal intermittent hypoxia only in women.

Conclusions: Nocturnal intermittent hypoxia was highly prevalent among T2DM patients and may be an independent associated risk factor for microalbuminuria in Japanese women with T2DM.
\end{abstract}

European Journal of Endocrinology 169 239-246

\section{Introduction}

The prevalence of type 2 diabetes mellitus (T2DM) worldwide was $2.8 \%$ in 2000 and is estimated to increase to $4.4 \%$ by 2030 owing to an overwhelming increase in the obese population (1). The increase in the prevalence of T2DM is a serious burden to public health because this disease is not only a risk factor for macroand microvascular disorders but also associated with all-cause mortality (2). The current obesity epidemic has contributed to an increase in the prevalence of T2DM in Japan (3). T2DM is the leading cause of haemodialysis and blindness in Japan, and recent cohort studies have confirmed that T2DM is associated with an increased risk of coronary heart disease and all types of stroke, except haemorrhagic stroke (4).
The International Diabetes Federation has expressed the need for further research into the links between sleep-disordered breathing (SDB) and T2DM (5). Several cross-sectional studies have indicated that increases in the apnoea-hypopnoea index are independently associated with insulin resistance. In the Wisconsin Sleep Cohort Study conducted in a prospective longitudinal manner, the prevalence of DM was three to four times more in subjects with severe SDB than in those without SDB (6). However, SDB was not a causal factor in the development of T2DM after adjustment for age, sex and waist circumference (6). Meanwhile, nocturnal intermittent hypoxia, which is a surrogate marker of obstructive sleep apnoea (OSA), was reported to be associated with an increased risk for developing T2DM among middle-aged Japanese patients (7). 
Therefore, the role of SDB in the development of diabetes may differ among racial groups.

Studies on the association between SDB and microvascular complications among T2DM patients are limited, and only a few studies have investigated SDB in Japanese diabetic subjects. A relationship between severe SDB and diabetic retinopathy among 166 Japanese diabetic subjects was identified in a crosssectional case-control study (8). Meanwhile, Kashine et al. (9) investigated SDB and clinical factors in 40 Japanese diabetic subjects and found no association between SDB and complications of T2DM. The sample size of these previous studies was insufficient to identify relationships between SDB and complications of T2DM.

SDB is very common among patients with chronic kidney diseases $(10,11)$. Nocturnal hypoxia is associated with an increased risk of kidney function loss $(12,13)$, and short-duration continuous positive airway pressure (CPAP) therapy can be used for the treatment of microalbuminuria (14). However, the influence of nocturnal intermittent hypoxia on microalbuminuria among T2DM patients remains unknown.

In this study, we hypothesised that nocturnal intermittent hypoxia, as a surrogate marker of OSA, may be a potential and associated risk factor for microvascular complications. The aims of this study were to estimate the prevalence of nocturnal intermittent hypoxia among patients with T2DM and to examine the association between nocturnal intermittent hypoxia and microvascular diseases.

\section{Subjects and methods}

\section{Study population}

The Dogo Study is a multicentre prospective cohort study that recruited 513 Japanese patients with previously diagnosed T2DM (median age at recruitment, 62.0 years; range, $20-85$ years; men, 292 and women, 221) whose sleep pattern was assessed between September 2009 and December 2010. The Dogo Study Group consists of medical doctors who specialise in DM at ten hospitals in Ehime prefecture, Japan. T2DM was diagnosed according to the Japan Diabetes Society criteria (15). This study protocol received ethical approval from the institutional review board of Ehime University, functioning according to the 3rd edition of the Guidelines on the Practice of Ethical Committees in Medical Research issued by the Royal College of Physicians of London. Written informed consent was obtained from all patients after full explanation of the purpose and nature of all procedures used.

\section{Measurements}

All participants completed self-administered questionnaires about weight at the age of 20 years, the duration of diabetes, alcohol intake per day, numbers of cigarettes smoked per day, use of antihypertensive medication, use of antihyperlipidaemic medication and the Epworth sleepiness scale (ESS, eight items with a value of 0-3 for each and a total score of 0-24) (16). BMI was calculated as weight $(\mathrm{kg})$ divided by square of height in metres $\left(\mathrm{m}^{2}\right)$. Weight change since the age of 20 years was estimated using the current body weight and selfreported body weight at the age of 20 years.

Patients who smoked $\geq 1$ cigarette/day were regarded as current smokers. ESS scores $\geq 11$ were considered to represent excessive daytime sleepiness, as shown by previous studies $(16,17)$. Blood pressure was measured with a cuff in the sitting position after a rest period of more than $5 \mathrm{~min}$. Hypertension was defined as a systolic blood pressure of more than $140 \mathrm{mmHg}$ or a diastolic blood pressure of more than $90 \mathrm{mmHg}$, or both, or if the patients were already receiving antihypertensive drugs. Hyperlipidaemia was defined as a serum total cholesterol concentration of $>5.69 \mathrm{mmol} / \mathrm{l}$, a triglyceride concentration of $>1.69 \mathrm{mmol} / \mathrm{l}$ or a HDL-cholesterol concentration of $<1.03 \mathrm{mmol} / \mathrm{l}$, or if the patients were already being treated with lipid-lowering agents. Stroke and ischaemic heart disease were assessed using the results of selfadministered questionnaires, medical records and admission data.

\section{Assessments of complications of T2DM}

The complications of microvascular disease in T2DM are retinopathy, nephropathy and neuropathy; these complications were assessed using the following definitions. The diagnosis of retinopathy was indicated by the presence of haemorrhages, microaneurysms, soft and hard exudates, areas of neovascularisation or laser coagulation scars in at least one eye using florescence fundoscopy on dilated pupils within 3 months of recruitment. Several ophthalmology specialists checked the fundus of all participants. All ophthalmologists were blinded to the diagnosis of nocturnal intermittent hypoxia. Nephropathy was defined using the urinary albumin:creatinine ratio (UACR) to classify the participants as follows: normoalbuminuria, $<3.4 \mathrm{mg} / \mathrm{mmol}$ creatinine; microalbuminuria, $\geq 3.4 \mathrm{mg} / \mathrm{mmol}$ creatinine and nephropathy, $\geq 34 \mathrm{mg} / \mathrm{mmol}$ creatinine. UACR was calculated using the urine sample taken on the first morning (18). Diabetic neuropathy was defined if the patients showed two or more of the following three characteristics: neuropathic symptoms, the absence of ankle tendon reflexes and abnormal vibration perception threshold scores assessed with a $128 \mathrm{~Hz}$ tuning fork (19).

\section{Assessments of nocturnal intermittent hypoxia}

A pulse oximeter (PULSOX-3Si; Minolta Co., Osaka, Japan) was attached to the left wrist during one night 
of sleep. The sensor probe was fitted to the ring finger and secured with a tape by each subject. The internal memory of this device stores the values of blood oxygen saturation by performing a moving average for the last $5 \mathrm{~s}$, updated every second; the sampling time was short in order to avoid underestimation of oxygen desaturation (20). Data were downloaded to a personal computer via an interface (PULSOX IF-3; Minolta Co.) and were analysed using proprietary software supplied with the equipment (DS-3 version. 2.0a; Minolta Co.). We used the 3\% oxygen desaturation index (ODI) as an indicator of nocturnal intermittent hypoxia. The value of 3\% ODI was taken as the mean value over a period of sleep of at least $4 \mathrm{~h}$, as estimated by pulse oximetry. The severity of nocturnal intermittent hypoxia was defined by the $3 \%$ ODI level: normal, less than five events per hour; and nocturnal intermittent hypoxia, five events or more per hour $(21,22)$.

\section{Statistical analysis}

All numerical variables are expressed as mean \pm s.D. Statistical analysis was conducted using one-way ANOVA. We used multivariate logistic regression models to estimate the odds ratio (OR) for the presence of microalbuminuria, nephropathy, retinopathy and neuropathy. All statistical analyses were performed using JMP 9 (SAS Institute, Inc., Cary, NC, USA). All probability values for statistical tests were two tailed, and $P$ values of $<0.05$ were regarded as statistically significant.

\section{Results}

As shown in Table 1, the prevalence of nocturnal intermittent hypoxia was $45.4 \%$ among patients with T2DM. The group of patients with nocturnal intermittent hypoxia was older and had a higher BMI, a greater weight change since the age of 20 years, a higher smoking rate and an increased prevalence of hypertension, hyperlipidaemia, microalbuminuria and macroalbuminuria. Similarly, significant differences were observed between men in the nocturnal intermittent hypoxia group and the normal group in terms of BMI, weight change since the age of 20 years, smoking rate, hypertension, microalbuminuria and macroalbuminuria. Meanwhile, significant differences were also found between women in the nocturnal intermittent hypoxia group and the normal group in regard to age, BMI, weight change since the age of 20 years, creatinine, hypertension, hyperlipidaemia, microalbuminuria and macroalbuminuria.

Table 2 shows the relationship between nocturnal intermittent hypoxia and microvascular complications. In the sex- and age-adjusted model for ODI $\geq 5$ events/ $h$ (model 1), only the ORs for nephropathy (OR, 3.25; 95\% CI, 1.67-6.69) and microalbuminuria (OR, 2.18; 95\% CI, 1.49-3.20) showed a positive association, whereas retinopathy and neuropathy did not. After adjustment for sex, age, BMI, hypertension and hyperlipidaemia for ODI $\geq 5$ events/h (model 2), the ORs for nephropathy (OR, 2.84; 95\% CI, 1.38-6.18) and microalbuminuria (OR, 1.76; 95\% CI, 1.16-2.69) were significant. After adjustment for sex, age, BMI, hypertension, hyperlipidaemia, current smoking, current drinking, stroke, ischaemic heart disease, duration of T2DM and HbAlc (model 3), the multivariate analysis identified ODI $\geq 5$ events/h to be associated with microalbuminuria (OR, 1.84; 95\% CI, 1.16-2.96) and nephropathy (OR, 2.97; 95\% CI, 1.36-6.90). Microalbuminuria (OR, 1.64; 95\% CI, 1.11-2.68) and nephropathy (OR, 2.62; 95\% CI, 1.12-6.68) derived from model 1 showed an association with ODI $\geq 5$ events/h in men, but these associations were negated in models 2 and 3. By contrast, microalbuminuria (model 1: OR, 3.41; 95\% CI, 1.86-6.40; model 2: OR, 3.69; 95\% CI, 1.85-7.59 and model 3: OR, 3.12; 95\% CI, 1.45-6.95) and nephropathy (model 1: OR, 4.51; 95\% CI, 1.58-15.1; model 2: OR, 7.31; 95\% CI, 2.11-31.6 and model 3: OR, 5.23; 95\% CI, 1.45-23.8) were derived as factors from all three models and constantly associated with nocturnal intermittent hypoxia in women. However, retinopathy and neuropathy did not show any associations with nocturnal intermittent hypoxia in both sexes.

Table 3 shows the diabetic nephropathy ODI $\geq 5$ events/h ORs according to the duration of T2DM and the weight change since the age of 20 years. The ODI $\geq 5$ events/h OR for microalbuminuria was 1.94 (95\% CI, 1.12-3.39) for those patients with diabetes for a duration $<11$ years and $2.69(95 \%$ CI, 1.57-4.68) for those with diabetes $\geq 11$ years. Similarly, the ODI $\geq 5$ events/h OR for nephropathy was 2.69 (95\% CI, 1.76-11.1) among those with a duration of $\geq 11$ years. The OR for the association between weight change since the age of 20 years and microalbuminuria were significantly high in the following categories: weight change $\geq 0 \mathrm{~kg}(\mathrm{OR}, 2.57 ; 95 \% \mathrm{CI}, 1.29-5.29)$ and $\geq 10 \mathrm{~kg}$ (OR, 2.27; 95\% CI, 1.17-4.72), while the association between weight change since the age of 20 years and diabetic nephropathy were only observed in the category of weight change $\geq 0 \mathrm{~kg}$ (OR, 7.79; 95\% CI, 1.87-53.3). Table 3 shows ODI $\geq 5$ events/h OR for microalbuminuria and diabetic nephropathy among patients with T2DM in men. No differences were observed between the categories for the duration of T2DM and weight change since the age of 20 years. Table 3 shows ODI $\geq 5$ events/h OR for microalbuminuria and diabetic nephropathy among patients with $\mathrm{T} 2 \mathrm{DM}$ in women. The ODI $\geq 5$ events/h OR for microalbuminuria were 2.33 (95\% CI, 1.06-5.24) and 6.02 (95\% CI, 2.26-17.9) among patients with T2DM of a duration of $<11$ and $\geq 11$ years respectively. Similarly, the ODI $\geq 5$ events/h OR for nephropathy was 3.76 (95\% CI, 2.21-31.0) among those with a duration of $\geq 11$ years. The OR for the association between weight 


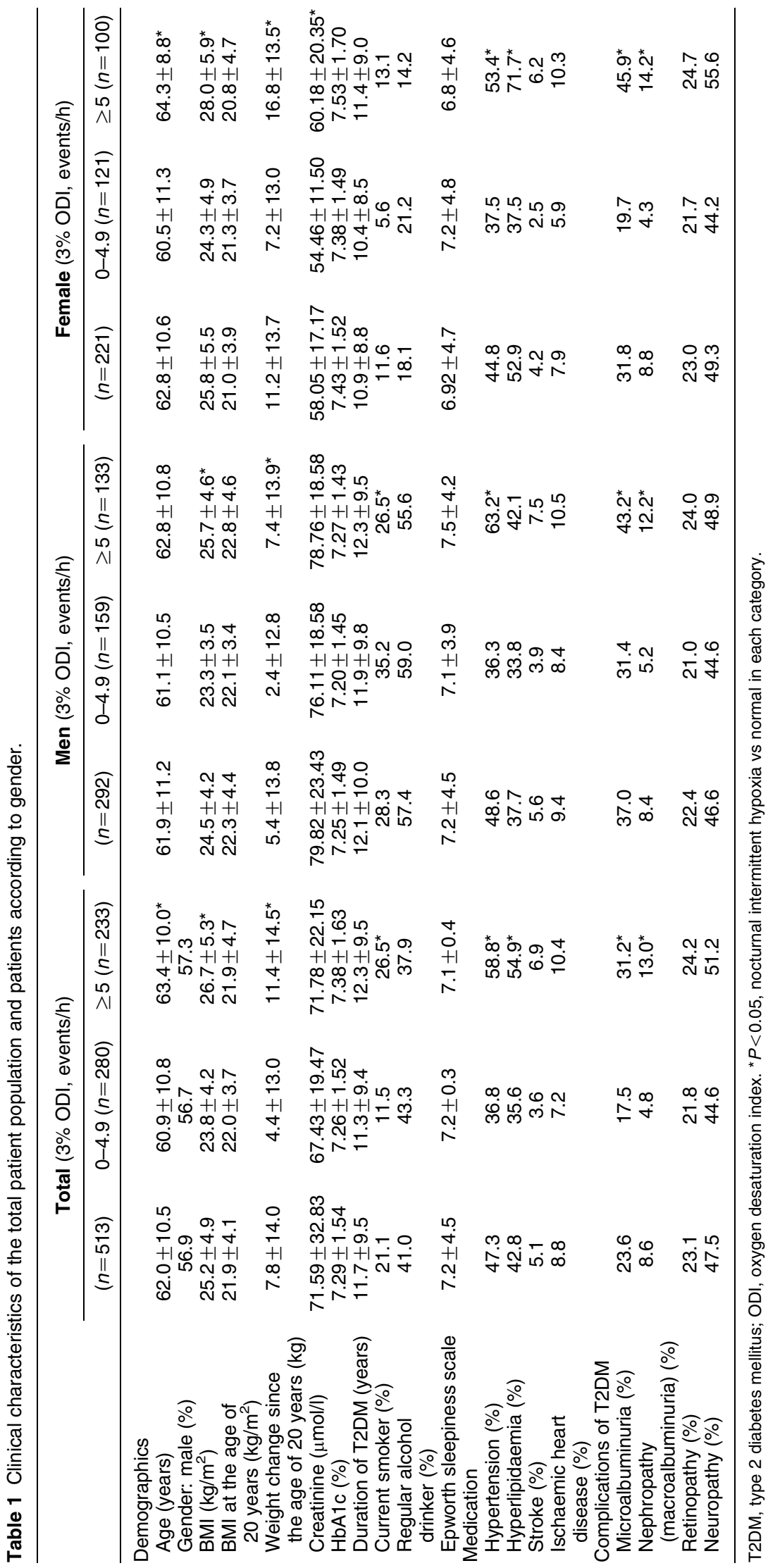


Table 2 The relationship between nocturnal intermittent hypoxia (ODI $\geq 5$ events/h) and microvascular complications according to gender and the total patient population. Model 1 was adjusted for sex and age (years). Model 2 was adjusted for factors cited above and BMl $\left(\mathrm{kg} / \mathrm{m}^{2}\right)$, hypertension and hyperlipidaemia. Model 3 was multivariable-adjusted for sex, age, BMI, hypertension, hyperlipidaemia, smoking status (current or not), drinking status (regular or not), current medications for stroke, ischaemic heart disease, duration of type 2 diabetes mellitus (years) and $\mathrm{HbA} 1 \mathrm{c}(\%)$.

\begin{tabular}{lcccc}
\hline & & ODI $\geq \mathbf{5}$ events/h OR $(95 \% \mathrm{Cl})$ \\
\cline { 2 - 5 } & Microalbuminuria & $\begin{array}{c}\text { Nephropathy } \\
\text { Macroalbuminuria }\end{array}$ & Retinopathy & Neuropathy \\
\hline Total $(n=513)$ & & & \\
$\quad$ Model 1 & $2.18^{*}(1.49-3.20)$ & $3.25^{*}(1.67-6.69)$ & $1.14(0.75-1.74)$ & $1.23(0.86-1.77)$ \\
Model 2 & $1.76^{*}(1.16-2.69)$ & $2.84^{*}(1.38-6.18)$ & $1.10(0.70-1.74)$ & $1.16(0.78-1.71)$ \\
Model 3 & $1.84^{*}(1.16-2.96)$ & $2.97^{*}(1.36-6.90)$ & $1.00(0.60-1.68)$ & $1.17(0.76-1.80)$ \\
Men $(n=292)$ & $1.64^{*}(1.11-2.68)$ & $2.62^{*}(1.12-6.68)$ & $1.14(0.75-1.74)$ & $1.10(0.67-1.78)$ \\
Model 1 & $1.03(0.58-1.83)$ & $1.57(0.60-4.31)$ & $1.04(0.57-1.89)$ & $0.85(0.50-1.44)$ \\
Model 2 & $1.17(0.62-2.20)$ & $1.90(0.63-6.22)$ & $0.87(0.44-1.73)$ & $0.84(0.45-1.53)$ \\
Model 3 & $3.41^{*}(1.86-6.40)$ & $4.51^{*}(1.58-15.1)$ & $1.19(0.63-2.27)$ & $1.49(0.86-2.58)$ \\
Female $(n=221)$ & $3.69^{*}(1.85-7.59)$ & $7.31^{*}(2.11-31.6)$ & $1.12(0.54-2.36)$ & $1.38(0.74-2.60)$ \\
Model 1 & $3.12^{*}(1.45-6.95)$ & $5.23^{*}(1.45-23.8)$ & $1.18(0.51-2.74)$ & $1.39(0.69-2.81)$ \\
Model 2 & & & &
\end{tabular}

OR, odds ratio. ${ }^{\star} P<0.05$ compared with reference category.

change since the age of 20 years and microalbuminuria were significant in the following categories: weight change $\geq 0 \mathrm{~kg}$ (OR, 6.00; 95\% CI, 1.82-21.9) and $\geq 10 \mathrm{~kg}(\mathrm{OR}, 3.68 ; 95 \% \mathrm{CI}, 1.52-9.70)$. Similarly, the OR for the association between weight change since the age of 20 years and nephropathy were significant in the following categories: weight change $\geq 0 \mathrm{~kg}$ (OR, 6.20; 95\% CI, 1.12-114.2) and $\geq 10 \mathrm{~kg}$ (OR, 6.99; 95\% CI, 1.21-133.0).

\section{Discussion}

Our results show that nocturnal intermittent hypoxia, defined as $3 \%$ ODI $\geq 5$ events/h, was associated with the presence of diabetic nephropathy among 513 patients with T2DM. The prevalence of nocturnal intermittent hypoxia among patients with T2DM was $45.5 \%$ in this study $(45.5 \%$ in men and $45.2 \%$ in women). Although we did not have a control group to assess the precise prevalence of nocturnal intermittent hypoxia among patients with T2DM, this group of subjects is likely to have higher nocturnal intermittent hypoxia than the general population.

An association between insulin resistance and microalbuminuria in T2DM has often been found in cross-sectional studies. In Taiwan, insulin resistance was reported as a predictive marker for the development of microalbuminuria in patients with T2DM (23). SDB is thought to cause insulin resistance via the following three steps. First, reduced oxygen desaturation stimulates sympathetic nerves. This stimulation is mediated by peripheral arterial chemoreceptors, particularly carotid bodies, with enhanced sympathetic drive apparently persisting during normal waking hours (24). Enhanced sympathetic drive raises serum catecholamine levels, leading to elevated serum glucose levels (25). Secondly, elevated oxidative stress raises cytokine levels (26), leading to increased insulin resistance (27). Thirdly, sleep fragmentation has been found to activate the hypothalamic-pituitary-adrenal axis (28), resulting in a marked elevation in serum cortisol levels, thereby leading to insulin resistance (29). Furthermore, chronic hypoxia has been identified as a key regulator in diabetic nephropathy (30), which is associated with the activation of hypoxia-inducible factor $1 \alpha(\mathrm{HIF} 1 \alpha)$. HIF 1 a plays an important role in diabetic nephropathy $(31,32,33)$, showing higher expression in diabetic kidneys than in normal human kidneys (34), and it has been reported as a novel target for the remission of diabetic nephropathy (35). Of interest, cell culture experiments have shown the upregulation of HIF $1 \alpha$ in intermittent hypoxia, while elevated serum levels of HIF1 gene products, such as EPO and vascular endothelial growth factor, have been demonstrated in OSA syndrome patients, particularly those with severe nocturnal hypoxaemia $(36,37,38,39)$.

According to our results, the relationship between other complications of T2DM and nocturnal intermittent hypoxia was not significant. Previous studies showed that lower BMI was a risk factor for retinopathy $(40,41)$, while nocturnal intermittent hypoxia showed a strong relationship with obesity. Therefore, the influence of nocturnal intermittent hypoxia on diabetic retinopathy may be weak. However, the mechanism underlying the association between lower BMI and diabetic retinopathy is unclear. The mean duration of T2DM in our population was more than 11 years, and therefore, the prevalence of diabetic neuropathy in our study was high. The association between nocturnal intermittent hypoxia and neuropathy may not be significant. 
Table 3 Sex- and age-adjusted ORs for microalbuminuria and diabetic nephropathy and nocturnal intermittent hypoxia stratified by the duration of T2DM and weight change since the age of 20 years in the total patient population and in male and female subjects.

\begin{tabular}{|c|c|c|c|}
\hline Stratification & $\begin{array}{l}\text { Number of } \\
\text { patients }\end{array}$ & $\begin{array}{c}\text { Microalbuminuria, } \\
\text { ODI } \geq \mathbf{5} \text { events } / \mathbf{h}, \\
\text { sex- and age-adjusted } \\
\text { OR }(95 \% \mathrm{Cl})\end{array}$ & $\begin{array}{c}\text { Nephropathy, } \\
\text { ODI } \geq \mathbf{5} \text { events/h, } \\
\text { sex- and age-adjusted } \\
\text { OR }(95 \% \mathrm{Cl})\end{array}$ \\
\hline \multicolumn{4}{|l|}{ Total $(n=513)$} \\
\hline \multicolumn{4}{|c|}{ Duration of T2DM (years) } \\
\hline$<11$ & 265 & $1.94^{\star}(1.12-3.39)$ & $3.11(0.99-11.73)$ \\
\hline$\geq 11$ & 248 & $2.69 *(1.57-4.68)$ & $2.69^{*}(1.76-11.1)$ \\
\hline \multicolumn{4}{|c|}{ Weight change since 20 years $(\mathrm{kg})$} \\
\hline$<0$ & 139 & $1.65(0.76-3.59)$ & $3.30(0.97-11.9)$ \\
\hline$\geq 0$ & 187 & $2.57^{*}(1.29-5.29)$ & $7.79^{*}(1.87-53.3)$ \\
\hline$\geq 10$ & 187 & $2.27^{\star}(1.17-4.72)$ & $2.7(0.91-1.88)$ \\
\hline \multicolumn{4}{|c|}{ Male $(n=292)$} \\
\hline \multicolumn{4}{|c|}{ Duration of T2DM (years) } \\
\hline$<11$ & 143 & $1.62(0.75-3.55)$ & $3.57(0.92-17.4)$ \\
\hline$\geq 11$ & 149 & $1.83(0.94-3.57)$ & $2.11(0.79-5.96)$ \\
\hline \multicolumn{4}{|c|}{ Weight change since 20 years $(\mathrm{kg})$} \\
\hline$<0$ & 102 & $1.84(0.78-4.37)$ & $5.87^{\star}(1.40-32.0)$ \\
\hline$\geq 0$ & 98 & $1.57(0.64-3.85)$ & $2.70(0.49-20.2)$ \\
\hline$\geq 10$ & 92 & $1.59(0.67-3.87)$ & $1.14(0.24-5.09)$ \\
\hline \multicolumn{4}{|c|}{ Female $(n=221)$} \\
\hline \multicolumn{4}{|c|}{ Duration of T2DM (years) } \\
\hline$<11$ & 122 & $2.33^{\star}(1.06-5.24)$ & $1.86(0.49-7.77)$ \\
\hline$\geq 11$ & 99 & $6.02 *(2.26-17.9)$ & $3.76 *(2.21-31.0)$ \\
\hline \multicolumn{4}{|c|}{ Weight change since 20 years $(\mathrm{kg})$} \\
\hline$<0$ & 37 & $0.56(0.02-4.48)$ & $2.10(0.76-4.12)$ \\
\hline$\geq 0$ & 89 & $6.00^{*}(1.82-21.9)$ & $6.20^{*}(1.12-114.2)$ \\
\hline$\geq 10$ & 95 & $3.68^{*}(1.52-9.70)$ & $6.99^{*}(1.21-133.0)$ \\
\hline
\end{tabular}

Table 3 shows that nocturnal intermittent hypoxia leads to microalbuminuria and diabetic nephropathy in patients with long-standing T2DM. Furthermore, the impact of nocturnal intermittent hypoxia was worse in obese patients. Moreover, this finding was rather significant in female patients. Long-term weight gain from adulthood to middle age increases the risk of T2DM in Japanese men and women. However, the risk of T2DM is further enhanced by weight gain in later life only in women (42). This may explain why nocturnal intermittent hypoxia affects microalbuminuria only in women. Meanwhile, hypertension may be a stronger risk factor for microalbuminuria in men as reported previously (43). To facilitate the remission and regression of microalbuminuria, it is important to perform CPAP therapy in the early stage of diabetic nephropathy and maintain adequate levels of plasma glucose and a healthy weight.

We acknowledge that our research had several limitations in terms of the study design. First and most importantly, our study did not have a control group to compare the prevalence of nocturnal intermittent hypoxia. However, the prevalence of nocturnal intermittent hypoxia in community-based Japanese men and women is reported to be $37.7-45.3 \%$ and $17.7-19.5 \%$ respectively (7). These results indicate that the prevalence of nocturnal intermittent hypoxia among T2DM may be higher than that in non-diabetic individuals.
Secondly, our study was cross-sectional, and we used a portable screening device to assess nocturnal intermittent hypoxia. Polysomnography is the gold standard for diagnosing OSA and is useful for evaluating nocturnal intermittent hypoxia (44). However, polysomnography is expensive, time-consuming and unsuitable for screening. Hence, we used reliable pulse oximetry to estimate nocturnal intermittent hypoxia (21). The validity of the pulse oximetry was confirmed by synchronous overnight recording of both PULSOX3Si and standard polysomnography among 256 consecutive patients who had been followed at an SDB centre in Japan (21). However, we were able to measure ODI only on one night at the patient's home and could not evaluate the ODI during the same day of the week. Finally, we obtained data on the weight change from 20 years of age from questionnaires.

Further, we were unable to perform renal biopsies for the diagnosis of diabetic nephropathy in all patients for ethical reasons. Finally, most of the participants had already been treated for diabetes for several years. Nevertheless, our data suggest that T2DM female patients with microalbuminuria undergo SDB screening and that nocturnal intermittent hypoxia should be regarded as a target to prevent end-stage renal disease, regardless of symptoms.

In conclusion, nocturnal intermittent hypoxia may be an independent associated risk factor for 
microalbuminuria in Japanese women with T2DM. Our study is the first report to show the relationship between nocturnal intermittent hypoxia and renal function among T2DM patients. Further longitudinal research is necessary to determine the relationship between nocturnal intermittent hypoxia and diabetic nephropathy and to elucidate the effects of CPAP on diabetic nephropathy.

\section{Declaration of interest}

The authors declare that there is no conflict of interest that could be perceived as prejudicing the impartiality of the research reported.

\section{Funding}

This study was supported in part by grants-in-aid for young scientists (B; grant number 217090583, 2008-2010) provided to S Furukawa from the Japanese Society for the Promotion of Science.

\section{Author contribution statement}

S Furukawa, S Yamamoto, T Miyake, T Niiya, T Ueda, M Torisu, T Sakai, H Minami, H Miyaoka, S Sakurai and B Matsuura conducted the research; S Furukawa, T Kumagi, I Saito and T Tanigawa reviewed and edited the manuscript and M Onji reviewed the manuscript.

\section{Acknowledgements}

The authors thank Drs Taiki Shimizu (Ehime University), Eriko Kawamoto (Matsuyama City Hospital), Shozo Miyauchi (Uwajima City Hospital) and Drs Eri Furukawa, Kentaro Ikeda and Ms Keiko Kikuchi (Saiseikai Matsuyama Hospital) for their assistance in assembling the data.

\section{References}

1 Wild S, Roglic G, Green A, Sicree R \& King H. Global prevalence of diabetes: estimates for the year 2000 and projections for 2030 . Diabetes Care 200427 1047-1053. (doi:10.2337/diacare.27. 5.1047)

2 Emering Risk Factors Collaboration, Seshasai SR, Kaptoge S, Thompson A, Di Angelantonio E, Gao P, Sarwar N, Whincup PH, Mukamal KJ, Gillum RF et al. Diabetes mellitus, fasting glucose, and risk of cause-specific death. New England Journal of Medicine 2011364 829-841. (doi:10.1056/NEJMoa1008862)

3 Kawamori R. Diabetes trends in Japan. Diabetes/Metabolism Research and Reviews 200218 (Suppl 3) S9-S13. (doi:10.1002/ dmrr.296)

4 Saito I. Epidemiological evidence of type 2 diabetes mellitus, metabolic syndrome, and cardiovascular disease in Japan. Circulation Journal 201276 1066-1073. (doi:10.1253/circj.CJ11-1519)

5 Shaw JE, Punjabi NM, Wilding JP, Alberti KG, Zimmet PZ \& International Diabetes Federation Taskforce on Epidemiology and Prevention. Sleep-disordered breathing and type 2 diabetes: a report from the International Diabetes Federation Taskforce on Epidemiology and Prevention. Diabetes Research and Clinical Practice 200881 2-12. (doi:10.1016/j.diabres.2008.04.025)

6 Reichmuth KJ, Austin D, Skatrud JB \& Young T. Association of sleep apnea and type II diabetes: a population-based study. American Journal of Respiratory and Critical Care Medicine 2005 172 1590-1595. (doi:10.1164/rccm.200504-6370C)

7 Muraki I, Tanigawa T, Yamagishi K, Sakurai S, Ohira T, Imano H, Kitamura A, Kiyama M, Sato S, Shimamoto T et al. Nocturnal intermittent hypoxia and the development of type 2 diabetes: the Circulatory Risk in Communities Study (CIRCS). Diabetologia 2010 53 481-488. (doi:10.1007/s00125-009-1616-0)

8 Shiba T, Sato Y \& Takahashi M. Relationship between diabetic retinopathy and sleep-disordered breathing. American Journal of Ophthalmology 2009147 1017-1021. (doi:10.1016/j.ajo.2008. 12.027)

9 Kashine S, Kishida K, Funahashi T, Nakagawa Y, Otuki M, Okita K, Iwahashi $\mathrm{H}$, Kihara S, Nakamura T, Matsuzawa $\mathrm{Y}$ et al. Characteristics of sleep-disordered breathing in Japanese patients with type 2 diabetes mellitus. Metabolism 201059 690-696. (doi:10.1016/j.metabol.2009.08.025)

10 Sakaguchi Y, Shoji T, Kawabata H, Niihata K, Suzuki A, Kaneko T, Okada N, Isaka Y, Rakugi H \& Tsubakihara Y. High prevalence of obstructive sleep apnea and its association with renal function among nondialysis chronic kidney disease patients in Japan: a cross-sectional study. Clinical Journal of the American Society of Nephrology 20116 995-1000. (doi:10.2215/CJN.08670910)

11 Chou YT, Lee PH, Yang CT, Lin CL, Veasey S, Chuang LP, Lin SW, Lin YS \& Chen NH. Obstructive sleep apnea: a stand-alone risk factor for chronic kidney disease. Nephrology, Dialysis, Transplantation 201126 2244-2250. (doi:10.1093/ndt/gfq821)

12 Ahmed SB, Ronksley PE, Hemmelgarn BR, Tsai WH, Manns BJ, Tonelli M, Klarenbach SW, Chin R, Clement FM \& Hanly PJ. Nocturnal hypoxia and loss of kidney function. PLoS ONE 20116 e19029. (doi:10.1371/journal.pone.0019029)

13 Nicholl DD, Ahmed SB, Loewen AH, Hemmelgarn BR, Sola DY, Beecroft JM, Turin TC \& Hanly PJ. Declining kidney function increases the prevalence of sleep apnea and nocturnal hypoxia. Chest 2012141 1422-1430. (doi:10.1378/chest.11-1809)

14 Daskalopoulou EG, Liavvas C, Nakas CT, Vlachogiannis EG, Bouros D \& Dombros NV. Obstructive sleep apnoea syndrome promotes reversal albuminuria during sleep. Sleep \& Breathing 201115 589-597. (doi:10.1007/s11325-010-0408-6)

15 The Japan Diabetes Society. Japan Diabetes Society Guidelines for the Management of Diabetes Based on Scientific Evidences. Tokyo: Japan Diabetes Society, 2004.

16 Johns MW. A new method for measuring daytime sleepiness: the Epworth sleepiness scale. Sleep 199114 540-545.

17 Johns M \& Hocking B. Daytime sleepiness and sleep habits of Australian workers. Sleep 199720 844-849.

18 Gross JL, de Azevedo MJ, Silveiro SP, Canani LH, Caramori ML \& Zelmanovitz T. Diabetic nephropathy: diagnosis, prevention, and treatment. Diabetes Care 200528 164-176. (doi:10.2337/ diacare.28.1.164)

19 Yokoyama H, Kawai K, Ohishi M, Sone H \& Japan Diabetes Data Management Study Group . Familial predisposition to cardiovascular risk and disease contributes to cardiovascular risk and disease interacting with other cardiovascular risk factors in diabetes: implication for common soil (JDDM 14). Atherosclerosis 2008201 332-338. (doi:10.1016/j.atherosclerosis.2008.02. 025)

20 Clark JS, Votteri B, Ariagno RL, Cheung P, Eichhorn JH, Fallat RJ, Lee SE, Newth CJ, Rotman H \& Sue DY. Noninvasive assessment of blood gases. American Review of Respiratory Disease 1992145 220-232. (doi:10.1164/ajrccm/145.1.220)

21 Nakamata M, Kubota Y, Sakai K, Kinefuchi S, Nakayama H, Ohdaira T, Satoh M, Shinoda H \& Kohno M. The limitation of screening test for patients with sleep apnea syndrome using pulse oximetry. Journal of Japan Society for Respiratory Care $2003 \mathbf{1 2}$ 401-406.

22 Tanigawa T, Tachibana N, Yamagishi K, Muraki I, Umesawa M, Shimamoto T \& Iso H. Usual alcohol consumption and arterial oxygen desaturation during sleep. Journal of the American Medical Association 2004292 923-935. (doi:10.1001/jama. 292.8.923-b)

23 Hsu CC, Chang HY, Huang MC, Hwang SJ, Yang YC, Tai TY, Yang HJ, Chang CT, Chang CJ, Li YS et al. Association between insulin resistance and development of microalbuminuria in type 2 diabetes: a prospective cohort study. Diabetes Care 201134 982-987. (doi:10.2337/dc10-1718) 
24 Narkiewicz K, van de Borne PJ, Montano N, Dyken ME, Phillips BG \& Somers VK. Contribution of tonic chemoreflex activation to sympathetic activity and blood pressure in patients with obstructive sleep apnea. Circulation $1998 \quad \mathbf{9 7} \quad 943-945$. (doi:10.1161/01.CIR.97.10.943)

25 Nonogaki K. New insights into sympathetic regulation of glucose and fat metabolism. Diabetologia $2000 \mathbf{4 3}$ 533-549. (doi:10.1007/s001250051341)

26 Ciftci TU, Kokturk O, Bukan N \& Bilgihan A. The relationship between serum cytokine levels with obesity and obstructive sleep apnea syndrome. Cytokine 200428 87-91. (doi:10.1016/j.cyto. 2004.07.003)

27 Uysal KT, Wiesbrock SM, Marino MW \& Hotamisligil GS. Protection from obesity-induced insulin resistance in mice lacking TNF- $\alpha$ function. Nature $1997389610-614$. (doi:10.1038/39335)

28 Stamatakis KA \& Punjabi NM. Effects of sleep fragmentation on glucose metabolism in normal subjects. Chest 2010137 95-101. (doi:10.1378/chest.09-0791)

29 Follenius M, Brandenberger G, Bandesapt JJ, Libert JP \& Ehrhart J. Nocturnal cortisol release in relation to sleep structure. Sleep 1992 15 21-27.

30 Eckardt KU, Bernhardt WM, Weidemann A, Warnecke C, Rosenberger C, Wiesener MS \& Willam C. Role of hypoxia in the pathogenesis of renal disease. Kidney International. (Supplement) 2005 S46-S51. (doi:10.1111/j.1523-1755.2005.09909.x)

31 Nangaku M. Chronic hypoxia and tubulointerstitial injury: a final common pathway to end-stage renal failure. Journal of the American Society of Nephrology 200617 17-25. (doi:10.1681/ ASN.2005070757)

32 Haase VH. The VHL/HIF oxygen-sensing pathway and its relevance to kidney disease. Kidney International $2006 \mathbf{6 9}$ 1302-1307. (doi:10.1038/sj.ki.5000221)

33 Eckardt KU, Bernhardt W, Willam C \& Wiesener M. Hypoxiainducible transcription factors and their role in renal disease. Seminars in Nephrology 200727 363-372. (doi:10.1016/ j.semnephrol.2007.02.007)

34 Higgins DF, Kimura K, Bernhardt WM, Shrimanker N, Akai Y, Hohenstein B, Saito Y, Johnson RS, Kretzler M, Cohen CD et al. Hypoxia promotes fibrogenesis in vivo via HIF-1 stimulation of epithelial-to-mesenchymal transition. Journal of Clinical Investigation 2007117 3810-3820. (doi:10.1172/JCI30487)

35 Bruegge K, Jelkmann W \& Metzen E. Hydroxylation of hypoxiainducible transcription factors and chemical compounds targeting the HIF- $\alpha$ hydroxylases. Current Medicinal Chemistry 200714 1853-1862. (doi:10.2174/092986707781058850)

36 Yuan G, Nanduri J, Bhasker CR, Semenza GL \& Prabhakar NR. $\mathrm{Ca}^{2+} /$ calmodulin kinase-dependent activation of hypoxia inducible factor 1 transcriptional activity in cells subjected to intermittent hypoxia. Journal of Biological Chemistry $20052 \mathbf{2 8 0}$ 4321-4328. (doi:10.1074/jbc.M407706200)

37 Yuan G, Nanduri J, Khan S, Semenza GL \& Prabhakar NR. Induction of HIF-1 $\alpha$ expression by intermittent hypoxia: involvement of NADPH oxidase, $\mathrm{Ca}^{2+}$ signaling, prolyl hydroxylases, and mTOR. Journal of Cellular Physiology 2008217 674-685. (doi:10.1002/jcp.21537)

38 Cahan C, Deker MJ \& Strohl KP. Humoral correlates of sleep apena: erythropoietin and hypoxemia. Progress in Clinical and Biological Research 1990345 317-322.

39 Winnicki M, Shamsuzzaman A, Lanfranchi P, Accurso V, Olson E, Davison D \& Somers VK. Erythropoietin and obstructive sleep apnea. American Journal of Hypertension 200417 783-786. (doi:10.1016/j.amjhyper.2004.04.011)

40 Klein R, Klein BE, Moss SE, Davis MD \& DeMets DL. The Wisconsin epidemiologic study of diabetic retinopathy. III. Prevalence and risk of diabetic retinopathy when age at diagnosis is 30 or more years. Archives of Ophthalmology 1984102 527-532. (doi:10.1001/archopht.1984.01040030405011)

41 Wong TY, Cheung N, Tay WT, Wang JJ, Aung T, Saw SM, Lim SC, Tai ES \& Mitchell P. Prevalence and risk factors for diabetic retinopathy: the Singapore Malay Eye Study. Ophthalmology 2008 115 1869-1875. (doi:10.1016/j.ophtha.2008.05.014)

42 Nanri A, Mizoue T, Takahashi Y, Matsushita Y, Noda M, Inoue M, Tsugane S \& Japan Public Health Center-based Prospective Study Group. Association of weight change in different periods of adulthood with risk of type 2 diabetes in Japanese men and women: the Japan Public Health Center-Based Prospective Study. Journal of Epidemiology and Community Health 201165 1104-1110. (doi:10.1136/jech.2009.097964)

43 Muiesan ML, Ambrosioni E, Costa FV, Leonetti G, Pessina AC, Salvetti M, Trimarco B, Volpe M, Pontremoli R, Deferrari G et al. Sex differences in hypertension-related renal and cardiovascular diseases in Italy: the I-DEMAND study. Journal of Hypertension 201230 2378-2386. (doi:10.1097/HJH.Ob013e328359b6a9)

44 Rosechtschaffen A \& Kalea A. In A Manual of Standardized Terminology, Techniques and Scoring System for Sleep Stages of Human Subjects. Washington, DC: US Government Printing Office, 1968.

Received 19 July 2012

Revised version received 29 March 2013

Accepted 23 May 2013 